\title{
Les militants laïcs de l'Église réfractaire : le cas lyonnais
}

The Lay Militants of the Refractory Church during the Revolution: the Case of Lyon

\section{Paul Chopelin}

\section{(2) OpenEdition}

\section{Journals}

Édition électronique

URL : https://journals.openedition.org/ahrf/10745

DOI : 10.4000/ahrf.10745

ISSN : 1952-403X

Éditeur :

Armand Colin, Société des études robespierristes

Édition imprimée

Date de publication : 1 janvier 2009

Pagination : 159-182

ISBN : 978-2-200-92557-4

ISSN : 0003-4436

\section{Référence électronique}

Paul Chopelin, «Les militants laïcs de l'Église réfractaire : le cas lyonnais », Annales historiques de la Révolution française [En ligne], 355 | janvier-mars 2009, mis en ligne le 01 janvier 2012, consulté le 23 avril 2022. URL : http://journals.openedition.org/ahrf/10745 ; DOI : https://doi.org/10.4000/ahrf. 10745 


\title{
LES MILITANTS LAÏCS DE L'ÉGLISE RÉFRACTAIRE : LE CAS LYONNAIS
}

Paul CHOPELIN

\begin{abstract}
La Révolution voit s'affirmer la figure du militant politique, mais également celle du militant religieux, longtemps rejeté dans l'ombre à la fois par l'historiographie républicaine et par l'historiographie ecclésiastique. Dans leur opposition à la Constitution civile du clergé, les prêtres réfractaires trouvent pourtant le soutien de nombreux laïcs, qui investissent l'espace public pour défendre leur cause. L'étude du cas de Lyon permet de mettre en valeur les différentes formes de cet engagement militant. Dès avant 1789 , la ville abrite des associations catholiques laïques très bien structurées et fonctionnant sur le mode de la clandestinité. À partir du début de l'année 1791, elles servent de support à l'action des fidèles en faveur de l'Église réfractaire, manifestée principalement par l'opposition physique au clergé constitutionnel et à ses partisans, ainsi que par la distribution d'imprimés polémiques. Après 1792, leur engagement se limite presque exclusivement à la protection des prêtres proscrits. Par la suite, les congrégations laïques lyonnaises du $\mathrm{XIX}^{\mathrm{e}}$ siècle réemploieront avec succès les pratiques militantes apprises au cours de la Révolution.
\end{abstract}

Mots-clés : Lyon, clergé réfractaire, laïcs, militants.

Dans l'historiographie révolutionnaire, le terme de «militant » ou de «militantisme » est traditionnellement associé à la figure du sansculotte $^{1}$. Il est plus rarement utilisé pour qualifier les adversaires de la Révolution, même si le concept paraît tout aussi opérant dans ce cas. Quant au militantisme religieux, il n'est quasiment jamais évoqué par les

(1) Haim Burstin, L'invention du sans-culotte. Regards sur le Paris révolutionnaire, Paris, Odile Jacob, coll. du Collège de France, 2005, 234 p. 
historiens de la période. Le rôle des laïcs dans les conflits religieux de la Révolution a pourtant été mis en valeur depuis une trentaine d'années, mais les chercheurs ont avant tout travaillé sur les résistances - au clergé constitutionnel et à la déchristianisation de l'an II - et sur l'évolution des pratiques $^{2}$. L'Église réfractaire dispose néanmoins d'un important groupe de fidèles agissant en véritables militants du refus de la Constitution civile du clergé, s'opposant à la législation religieuse et ecclésiastique mise en œuvre de 1789 à 1798 et dépassant souvent le simple rôle de relais de l'opinion cléricale dans l'espace public.

Rappelons ici que le terme de «militant» est à l'origine un terme religieux. Il désigne l'action du chrétien dans sa vie terrestre, afin de résister aux tentations et de respecter les préceptes de l'Évangile : « La vie du chrétien est appelée militante, parce que la vie du chrétien sur la terre est regardée comme une milice, comme un combat qu'il doit livrer au monde, au démon et à ses propres passions $»^{3}$. Le mot militant tel qu'il est utilisé dans le présent article se comprend dans la version laïcisée et contemporaine du mot, apparue au XIX siècle, et qui désigne une personne cherchant à faire triompher ses idées ou à défendre une cause par l'action publique ou secrète. Cette définition n'entre pas en contradiction avec l'acception religieuse du terme : le militantisme analysé ici est une forme d'externalisation publique de la vie militante du chrétien. Le militant laïc de l'Église réfractaire est donc plus précisément compris comme une personne agissant par la parole, par l'écrit ou par le geste physique pour dénoncer la Constitution civile du clergé. Ce militant n'est pas forcément un ennemi de la Révolution, même si, au regard des autorités, il se range dans le camp des « contre-révolutionnaires » en s'opposant à la $1 \mathrm{i}^{4}$ : dans l'absolu, il refuse seulement la politique religieuse mise en œuvre par le pouvoir législatif à partir de 1790. Si certains de ces militants réfractaires rejettent en bloc la Révolution, d'autres font le tri entre les réformes politiques, que le bon chrétien, soumis aux puissances de la

(2) Un bon aperçu des recherches entreprises à ce sujet dans les années 1970 et 1980 à travers les $2^{\mathrm{e}}$ et $3^{\mathrm{e}}$ parties ( « vie religieuse et sacramentalisation des fidèles »; " résistances et mutations culturelles ») du colloque de Chantilly (1986) : Bernard Plongeron (dir.), Pratiques religieuses, mentalités et spiritualités dans l'Europe révolutionnaire (1770-1820), Turnhout, Brepols, 1988, p. 163-366.

(3) Abbé Nicolas-Sylvestre Bergier, Encyclopédie méthodique. Théologie. Tome second, Paris, Panckoucke, 1789, p. 643. L'Église chrétienne se compose ainsi de trois éléments : l'Église militante (la société des fidèles sur la terre), l’Église souffrante (les âmes des fidèles en purgatoire) et l'Église triomphante (la communion des saints).

(4) Jean-Clément Martin, Contre-Révolution, Révolution et Nation en France (1789-1799), Paris, Seuil, coll. Points Histoire, 1998, 367 p. 
terre, doit accepter, et les réformes religieuses qui posent un problème à sa conscience. Ils contestent donc le pouvoir des autorités locales qui mettent en application les décisions religieuses votées par les députés, sanctionnées (ou non) par le roi, et condamnées par le pape ou les autorités diocésaines. Le militant chrétien devient ainsi un militant politique. Il convient ici de distinguer le militant du simple fidèle. Si le militant est un fidèle du clergé réfractaire ${ }^{5}$, tous les fidèles ne sont pas des militants. Le fidèle assiste à la messe et reçoit les sacrements d'un prêtre réfractaire : il ne cherche pas forcément à être un prosélyte et à diffuser les arguments du clergé insermenté dans son entourage. On peut néanmoins nuancer cette opposition en rappelant que, lors de périodes de contestation du libre exercice du culte réfractaire, le fidèle qui se rend publiquement à la messe accomplit en soi un acte militant. Il s'agit alors d'une démarche publique, observée en présence de foules " patriotes », rassemblées devant les chapelles des «insoumis» pour conspuer ou agresser physiquement les fidèles : le simple fait de participer à la célébration du culte devient ainsi une confession de foi et peut prendre un caractère militant.

Parallèlement au militantisme révolutionnaire, s'organise donc une autre forme d'engagement, qui utilise les mêmes armes, notamment l'imprimé, pour convaincre le public de l'invalidité des réformes religieuses votées par les représentants de la Nation. Mais avant d'aborder le déploiement de cet activisme réfractaire dans l'espace urbain lyonnais, il convient d'inscrire certaines de ces pratiques militantes dans un temps long, largement antérieur à la Révolution.

\section{Les origines}

Le militantisme de ces laïcs catholiques pendant la Révolution prend ses racines dans les pratiques mises en œuvre à partir du XVII siècle par les sodalités dévotes de la Réforme catholique ${ }^{6}$. Symbole de ce militantisme tridentin, la Compagnie du Saint-Sacrement a profondément marqué le catholicisme lyonnais: présente dans la ville depuis 1630 , l'association a continué de recevoir des membres jusqu'en 1731, date à laquelle figure la dernière inscription sur son registre, soit plus de soixante

(5) Quoiqu'il faille ici aussi nuancer. Ainsi, les publicistes royalistes qui dénigrent la Constitution civile du clergé et dénoncent l'hétérodoxie des réformes religieuses mises en œuvre par l'Assemblée nationale entre 1790 et 1792 ne sont pas tous d'ardents pratiquants, loin de là.

(6) Louis Châtellier, L'Europe des dévots, Paris, Flammarion, Nouvelle Bibliothèque scientifique, 1987, 315 p. ; Jean-Pierre GutTon, Dévots et société au XVII siècle. Construire le Ciel sur la Terre, Paris, Belin, coll. Histoire \& Société, 2004, 220 p. 
ans après la dissolution officielle de la Compagnie par Louis XIV. Son influence a été déterminante dans la diffusion des principes tridentins dans la ville, en introduisant de nouvelles formes de dévotion ou en procédant à la christianisation de la société par le biais de l'action éducative et de l'assistance ${ }^{7}$. Les notables lyonnais membres de la Compagnie poursuivent leur action à travers d'autres structures, notamment les confréries de pénitents. Ainsi, les Pénitents de Notre-Dame du Confalon et les Pénitents de la Miséricorde, qui comptent dans leurs rangs toute l'élite politique et marchande de la ville, constituent très certainement l'un des principaux relais de la Compagnie au XVIII ${ }^{e}$ siècle. Leur action dans la cité est très importante à la veille de la Révolution, notamment lors de la crise économique qui touche l'activité textile à partir des années 1770. En 1771 et 1774, les Pénitents de Notre-Dame du Confalon distribuent des secours aux familles des ouvriers au chômage: plus de 20000 personnes auraient bénéficié de leurs largesses selon l'Almanach de Lyon. Lors de la grève de 1786, ils convertissent la rétribution des chaises de leur chapelle en aumônes pour les chômeurs.

Depuis la seconde moitié du XVII siècle, Lyon accueille également des Aas. Ces assemblées de piété, dont le nom serait soit une contraction d'Associatio Amicorum, soit l'abréviation d' « assemblées », sont de petits groupes de jeunes prêtres ou séminaristes - une vingtaine en général - qui partagent une même ferveur et une volonté commune d'agir concrètement, mais secrètement, sur la société, dans le cadre d'institutions religieuses d'assistance ou d'éducation. Ces groupes peuvent également se composer de laïcs - on parle d' $A a$ de Messieurs - poursuivant des objectifs similaires $^{8}$. Les sources concernant ces associations secrètes sont malheureusement très rares. Si le Toulousain est assez bien documenté, tel n'est pas le cas de Lyon. La première partie d'un registre d'une Aa lyonnaise a été conservée, retraçant son histoire de 1668 à $1732^{9}$. Son recrutement s'organise autour du séminaire Saint-Irénée, tenu par les sulpiciens, et du

(7) Georges Guigue (éd.), Les papiers des dévots de Lyon. Recueil de textes sur la Compagnie secrète du Saint-Sacrement, ses statuts, ses annales, la liste de ses membres (1630-1731), Lyon, Blot, 1922, XVII + 246 p. Alain Tallon, La Compagnie du Saint-Sacrement (1629-1667). Spiritualité et société, Paris, Cerf, 1990, 189 p.

(8) Robert RouQuetTe, « Congrégations secrètes », dans Dictionnaire de spiritualité, t. II, col. 1491-1505 ; Jacques Godechot, «Quel a été le rôle des Aa pendant l'époque révolutionnaire?», dans Mélanges offerts à Monsieur le doyen André Latreille, Lyon, Audin, 1972, p. 105-115.

(9) Yves Poutet et Jacqueline Roubert, Les "Assemblées » secrètes des XVII ${ }^{e}$-XVIII $I^{e}$ siècles en relation avec l'Aa de Lyon. Édition critique des Annales d'une Aa lyonnaise, Piacenza, Divus Thomas, 1968, $174 \mathrm{p}$. 
Grand Collège administré par les jésuites. Cette $A a$ mène une existence parallèle et distincte de la Compagnie du Saint-Sacrement, même si plusieurs membres finissent par adhérer à la Compagnie. Les renseignements font cruellement défaut pour la seconde moitié du XVIII siècle, mais il est fort probable que le séminaire Saint-Irénée accueille encore une ou plusieurs Aas en ses murs en 1789. La cohésion et l'esprit de corps dont font preuve les «philosophes », jeunes élèves laïcs venus au séminaire Saint-Irénée pour suivre une formation succincte en théologie à la fin de leurs études, laissent supposer l'existence d'une structure associative de type $A a$ de Messieurs. On observe effectivement une solidarité entre anciens "philosophes » et une volonté commune de défendre l’Église catholique face aux attaques de ses ennemis. Les affrontements religieux de la Révolution sont l'occasion de mettre en valeur cet engagement confraternel.

Une nouvelle forme d'association religieuse de laïcs voit également le jour dans Lyon à l'initiative d'un prêtre habitué de la collégiale SaintNizier, l'abbé Jacques Linsolas (1754-1828). Ce dernier est animé d'un ardent esprit missionnaire, qui le conduit à s'inscrire en 1788 au séminaire des Missions étrangères de Paris, avec le projet de participer à l'évangélisation du Tonkin et de la Cochinchine, mais il finit par renoncer et rentre à Lyon au cours de l'année 1789. En 1783, il avait organisé une « association de jeunes gens ", association de piété composée d'anciens catéchisés dont il avait remarqué le zèle pour la religion. Ces derniers sont amenés à approfondir leur éducation morale et religieuse par des exercices spirituels communs et des lectures édifiantes, destinées à les prémunir des séductions du libertinage et de l'incrédulité. Fort du succès de cette première œuvre, il crée en 1788 une "société des demoiselles », apparemment beaucoup plus structurée que la première association, selon les renseignements fournis par le fondateur lui-même dans ses mémoires :

« Il [Linsolas] choisit des personnes raisonnables, pieuses, appartenant à des familles honnêtes et chrétiennes ; il donna à cette société un règlement, qu'il adapta aux circonstances pénibles auxquelles il présumait qu'on serait exposé dans les jours malheureux qu'il prévoyait. Pour lui donner une certaine étendue dans le besoin, chaque associée devait s'affilier secrètement, et sans faire connaître l'association, quatre ou cinq parentes ou amies, pour les porter à la pratique de la vertu, principalement à la charité effective envers le prochain. Les associées et affiliées s'occupèrent, jusqu'à nouvel ordre, à édifier leur famille, à se porter mutuellement, avec une sainte émulation, à la piété et aux bonnes œuvres. Toutes 
les trois semaines, il y avait une assemblée pour toutes les associées, et chaque membre de cette société réunissait tous les mois au moins ses affiliés $»^{10}$.

Cette association suit le même mode de fonctionnement qu'une $A a$. Cette structure pyramidale et cloisonnée, dans laquelle les affiliées ignorent tout de la société, rappelle également la Compagnie du SaintSacrement.

Avant la Révolution, ces sociétés dévotes entretiennent somme toute un militantisme dévot traditionnel, visant à christianiser la société par l'éducation et les bonnes œuvres. En revanche, une nouvelle forme de militantisme catholique apparaît avec le jansénisme convulsionnaire : il s'agit non pas tant de christianiser que de convaincre le public de la justesse d'une cause. En cela les militants du mouvement convulsionnaire lyonnais apparaissent bien comme les véritables précurseurs du militantisme catholique de la période révolutionnaire. Ce mouvement dérivé du jansénisme apparaît à Lyon, à la fin des années 1720, autour de la figure du P. Céloron, un oratorien appelant, qui aurait guéri miraculeusement un enfant aveugle. Cet événement suscite l'enthousiasme de plusieurs laïcs lyonnais, qui assistent nombreux, en 1729, à l'enterrement du prêtre thaumaturge, dont les fidèles se disputent les reliques. Néanmoins, à l'inverse du cas parisien de Saint-Médard, ce miracle ne semble pas aboutir à la constitution d'un groupe organisé. Ce n'est qu'au début des années 1730 que la dévotion au diacre Pâris et ses manifestations surnaturelles atteignent Lyon, notamment lorsque les Nouvelles ecclésiastiques répandent la nouvelle de la guérison miraculeuse d'un ouvrier lyonnais par l'application de terre provenant du tombeau de Saint-Médard. Les autorités diocésaines lyonnaises combattent énergiquement et, semble-t-il, avec efficacité ces pratiques, car il faut attendre les années 1770 pour assister à la formation d'un groupe organisé de convulsionnaires dans la capitale des Gaules : l'CEuvre de Lyon. Le fonctionnement de cette association est connu par un ouvrage hostile, écrit par un prêtre janséniste adversaire des convulsions ${ }^{11}$. Une « Euvre » est un réseau de convulsionnaires, prêtres et laïcs, organisé au niveau d'un ou de plusieurs diocèses,

(10) Jacques Linsolas, L'Église clandestine de Lyon pendant la Révolution, Lyon, Éditions lyonnaises d'Art et d'Histoire, 1987, vol. 2, p. 110.

(11) [Pierre CREPE], Notion de l'œuvre des convulsions et des secours, surtout par rapport à ce qu'elle est dans nos provinces du Lyonnois, Forez, Mâconnois, etc., à l'occasion du crucifiement public de Fareins, s. 1. n. d. [1788], 304 p. 
qui correspond avec d'autres groupes à Paris et dans les provinces. L'Euvre de Lyon a établi son siège dans la ville de Lyon, mais rayonne sur le Forez et le Mâconnais, entretenant des correspondances avec tous les groupes du Midi, dont elle serait la « mère Église ». Chaque Euvre possède ses propres visionnaires, diffuse ou échange prophéties et récits de convulsions avec ses consœurs. À Lyon, la cheville ouvrière du mouvement convulsionnaire est un laïc, Claude-François Desfours de La Genetière (1758-1819). C'est un véritable militant, au sens contemporain du terme, passant le plus clair de son temps, grâce à sa fortune personnelle, à promouvoir la cause des convulsionnaires auprès du public lyonnais et forézien. Claude Hau l'avait surnommé avec justesse « le commis-voyageur » de l'CEuvre ${ }^{12}$. Se considérant comme un nouveau Carré de Montgeron ${ }^{13}$, Desfours organise des séances de convulsions dans son domaine de Grange-Blanche, dans les faubourgs ouest de la ville. Il recueille avec minutie les prophéties et les visions des convulsionnaires qu'il diffuse parmi les «Amis de la vérité $»^{14}$. En 1784, il publie une synthèse des visions et prophéties dont il a été le témoin et le propagateur: Les trois états de l'homme par rapport à la justice, ouvrage qui connaît plusieurs rééditions des années 1780 au milieu du XIX ${ }^{\mathrm{e}}$ siècle ${ }^{15}$. Mais ce militantisme s'efface pendant la Révolution : si Desfours et ses amis sont hostiles à la Constitution civile du clergé, ils s'enferment dans une logique sectaire et exclusive, se préparant à une fin du monde jugée imminente. Le crucifiement de deux adeptes, à Marcilly-le-Châtel et à Fareins en 1787, achève de déconsidérer l'Euvre au sein de la population lyonnaise. Les militants jansénistes ne cherchent dès lors plus guère à convertir, abandonnent l'espace public et se replient sur eux-mêmes, dans l'attente de la venue du prophète Élie ${ }^{16}$.

(12) Claude Hau, Le Messie de l'an XIII, Paris, Denoël, coll. Présence du Passé, 1955, 253 p.

(13) Pendant tout le XVIII siècle, Louis-Basile Carré de Montgeron (1686-1754) incarne le modèle du parfait militant janséniste. Il avait défendu la cause de manière spectaculaire en remettant son ouvrage la Vérité de miracles à Louis XV en personne, dans la galerie des glaces de Versailles, en juillet 1737. Cette action d'éclat lui valut un séjour à la Bastille, suivi d'un exil forcé. Il devient dès lors un martyr de la vérité, dont les épreuves sont relatées complaisamment dans les colonnes des Nouvelles ecclésiastiques. Sur ce personnage, Joseph Dedieu, «L'agonie du jansénisme (1715-1790). Essai de bio-bibliographie », dans Revue d'histoire de l'Église de France, t. XIV, n 63, 1928, p. 161-214.

(14) Jean-Pierre Chantin, « Claude-François Desfours de La Genetière », dans Xavier de Montclos (dir.), Dictionnaire du monde religieux dans la France contemporaine. Tome 6. Lyon, le Lyonnais, le Beaujolais, Paris, Beauchesne, 1994, p. 157 ; Daniel VIDAL, La Morte Raison. Isaac la Juive, convulsionnaire janséniste de Lyon (1791-1841), Grenoble, Jérôme Millon, 1994, 212 p.

(15) [Claude-François Desfours de LA GENETIÈRE], Les trois États de l'homme, par rapport à la justice ou L'Homme considéré avant la loi, sous la loi et sous la grâce, s. 1. [Lyon], 1784, 294 p.

(16) Jean-Pierre Chantin, Les Amis de l'Euvre de la Vérité. Jansénisme, miracles et fin du monde, Lyon, PUL, 1998, 184 p. 
Si les Amis de la vérité ne jouent plus qu'un rôle marginal à Lyon pendant la Révolution, il ne faut pas oublier que leur organisation a pu inspirer l'abbé Linsolas dans la mise en place de ses sociétés laïques. Dans les deux cas, l'efficacité du groupe repose sur le secret et sur un recrutement familial, propre à activer des réseaux de sociabilité locaux en faveur de la cause. Il est possible que Linsolas, témoin des progrès du mouvement convulsionnaire dans les milieux dévots lyonnais au cours des années 1770, ait voulu contrer les jansénistes sur leur propre terrain. Il est tout aussi probable qu'ayant constaté l'efficacité militante de l'Euvre de la vérité, Linsolas ait souhaité simplement imiter cette structure militante en organisant son réseau d'anciens catéchisés. En tout état de cause, lorsque commence la Révolution, des associations de fidèles catholiques sont déjà organisées à Lyon sur le mode de l'action clandestine.

\section{La lutte contre la Constitution civile du clergé (1790-1792)}

Le clergé réfractaire s'organise à Lyon au cours de l'année 1790. Le décret du 13 avril, par lequel les députés refusent de reconnaître à la religion catholique le statut de religion nationale, introduit un premier clivage parmi les prêtres et les fidèles lyonnais. La presse patriote, la municipalité et les sections invitent les ecclésiastiques à manifester publiquement leur soutien à l'Assemblée nationale, tandis que les prêtres hostiles sont dénoncés comme «traîtres à la nation ». Le 22 mai 1790, le terme de « prêtres réfractaires » est utilisé pour la première fois dans les colonnes du Courier de $\mathrm{Lyon}^{17}$. Le serment à la Constitution imposé aux prêtres exerçant un ministère public à partir de novembre 1790 accélère la division de l'Église catholique entre « constitutionnels » et « réfractaires », consommée par la condamnation pontificale de la Constitution civile du clergé en marsavril 1791. Les laïcs doivent choisir leur camp et affûtent leurs arguments afin de convaincre le plus grand nombre de leurs concitoyens.

Plusieurs confréries lyonnaises se déchirent à cette occasion. C'est le cas des Pénitents de Notre-Dame du Confalon. Lorsque le nouvel évêque constitutionnel de Rhône-et-Loire, Adrien Lamourette, élu le $1^{\text {er }}$ mars 1791, doit venir prêcher dans la chapelle de la confrérie le dimanche des Rameaux suivant, plusieurs confrères refusent de l'accueillir et sont exclus par la majorité des membres, favorables à l'Église constitutionnelle ${ }^{18}$. La Société des Hopitaliers-Veilleurs se scinde également en

(17) Courier de Lyon, t. V, n¹9, 22 mai 1790, p. 161.

(18) Journal de Lyon ou Moniteur du département de Rhône-et-Loire, $\mathrm{n}^{\circ} 16,9$ mai 1791, p. 3. 
deux groupes : les «constitutionnels », qui poursuivent leurs bonnes œuvres auprès des malades des hôpitaux lyonnais, encadrés par des prêtres constitutionnels, et les "réfractaires », qui ne s'occupent plus que du soulagement spirituel et matériel des pauvres prisonniers ${ }^{19}$. Il est difficile d'obtenir un tableau d'ensemble du choix opéré au sein des confréries lyonnaises en 1790-1791, du fait de l'absence de sources. Il est néanmoins possible de repérer quelques associations qui optent majoritairement pour le clergé réfractaire. La confrérie des Pénitents de la Miséricorde fait ainsi célébrer des messes dans leur chapelle par des prêtres insermentés, jusqu'à la fermeture des lieux ordonnée par la municipalité de Lyon au cours de l'été $1792^{20}$. Les Pénitents du saint Crucifix rejoignent eux aussi le camp réfractaire. Cet engagement s'explique très certainement par l'option des directeurs spirituels de la confrérie, qui se trouvent être les principaux chefs du clergé réfractaire lyonnais : le vicaire général Joseph Courbon, le chanoine-comte de Rully et l'abbé Linsolas ${ }^{21}$. Les associations laïques secrètes de ce même abbé Linsolas sont bien évidemment entraînées elles aussi par leur directeur dans le camp réfractaire.

Ces laïcs ne se contentent pas de prières ou d'actions charitables, mais investissent également l'espace public, pour défendre la cause de «l'Église légitime ». Les actions de ces militants peuvent se grouper en deux catégories : la propagande, par la diffusion de textes légitimant la position du clergé réfractaire, et des manifestations physiques d'opposition, dans les rues et les églises.

Il n'est plus à démontrer que la Révolution correspond à un grand moment de lecture, publique et privée, appuyé sur une abondante production imprimée de livres, journaux, brochures et images en tout genre. À l'instar de tous les acteurs de la période, le clergé réfractaire utilise l'arme de l'imprimé pour convaincre prêtres et fidèles de la justesse de sa cause. À Lyon, si la majorité des auteurs connus de ces textes sont des ecclésiastiques, quelques laïcs ont pu participer à la polémique, de façon anonyme. Camille Jordan est l'un d'eux. Né à Lyon en 1771, il passe par les classes de philosophie du séminaire Saint-Irénée dans la seconde moitié des années 1780 et devient probablement membre de l'Aa de Saint-Irénée. Il apporte son soutien au clergé réfractaire en rédigeant

(19) Dominique Meynis, Les Hospitaliers-Veilleurs. Société charitable fondée à Lyon en 1763. Notice historique, Lyon, Alricy, 1882, p. 15-19.

(20) Jacques Linsolas, L'Église clandestine de Lyon, op. cit., vol. 2, p. 65 et 81-82.

(21) BM Lyon, Fonds Coste, ms. 354 : registre de la confrérie des Pénitents du saint Crucifix de Lyon (1589-1590), ff $33-39$. 
plusieurs libelles anonymes diffusés à Lyon au début de la Révolution : une Lettre à M. Lamourette (1791), une Histoire de la conversion d'une dame parisienne (1792) et, sous le pseudonyme de Simon, La loi et la religion vengées des violences commises aux portes des églises catholiques de Lyon (1792) ${ }^{22}$. Si les laïcs sont rarement les auteurs des textes théologiques ou polémiques répandus contre la Constitution civile du clergé, ils prennent le plus souvent en charge leur impression et leur diffusion $^{23}$. À Lyon, la veuve Rusand est ainsi le principal maître d'œuvre de la propagande du clergé insermenté. Sise rue Mercière, sa librairie est spécialisée dans le livre religieux et bénéficie d'une importante clientèle ecclésiastique. À partir de 1790, elle soutient ostensiblement le clergé réfractaire, dont elle fait imprimer les textes ou revend clandestinement des brochures qui lui sont favorables. Au début du mois de mars 1791, la municipalité de Lyon saisit dans son fonds 500 exemplaires du dernier avertissement pastoral de $\mathrm{M}^{\mathrm{gr}}$ de Marbeuf, archevêque réfractaire de Lyon $^{24}$. Au mois d'avril suivant, c'est dans sa boutique que le clergé réfractaire et ses partisans viennent acheter des exemplaires du bref Quod Aliquantum pour le répandre en ville ${ }^{25}$. Cette activité ne l'empêche pas de vendre des livres au clergé constitutionnel, car on sait qu'elle fournit le séminaire métropolitain de Lyon en livres et en journaux patriotes ${ }^{26}$. On peut en effet observer que plusieurs imprimeurs-libraires lyonnais jouent sur les deux tableaux, en imprimant ou en diffusant des ouvrages favorables

(22) Le soutien actif apporté par Camille Jordan au clergé réfractaire en 1790-1792 a été dévoilé par ses amis après le décès de l'intéressé : Discours de Camille Jordan, précédés de son éloge par M. Ballanche, d'une lettre de M. le baron Degerando sur sa vie privée, suivis de fragmens inédits et des discours qui ont été prononcés sur sa tombe par MM. Royer-Collard et de Saint-Aulaire, et ornés de son portrait et d'un fac-simile de son écriture, Paris, Renouard, 1826, p. VI-VII ; Les références complètes des trois imprimés attribués à Jordan sont les suivantes : Lettre à M. Lamourette, se disant évêque de Rhône-et-Loire et métropolitain du Sud-Est, s. 1. n. d. [Lyon, 1791] ; Histoire de la conversion d'une dame parisienne, écrite par elle-même, Paris, Lallemand, 1792, 64 p. ; SimON, La loi et la religion vengées des violences commises aux portes des églises catholiques de Lyon, $\mathrm{s}$. 1. n. d. [Lyon, 1792], 16 p. Sur cette attribution, voir Joseph-Marie QuÉRARD, La France littéraire, Paris, Didot, 1830, t. IV, p. 245.

(23) Il faut signaler l'exception de l'abbé Colonjon, à Annonay, qui imprime des textes hostiles à l'Église constitutionnelle et les fait circuler dans toute la vallée du Rhône, ainsi que dans le Lyonnais.

(24) Procès-verbaux des séances des corps municipaux de la ville de Lyon, publiés par la municipalité d'après les manuscrits originaux (1787-an VII), Lyon, Imprimerie nouvelle, 1900, t. II, séance du 22 mars 1791, p. 361.

(25) Journal de Lyon ou Moniteur du département de Rhône-et-Loire, $\mathrm{n}^{\circ}$ 10, 23 avril 1791, p. 38 et $\mathrm{n}^{\circ} 16,9$ mai 1791, p. 3.

(26) AD Rhône, 1 Q 1190 : livre de comptes du séminaire métropolitain de Rhône-et-Loire (1791-1792). 
à l'un et l'autre camp. C'est le cas de Joseph-Sulpice Grabit ou des libraires associés Maire et Mars, inquiétés pour avoir vendu des imprimés hostiles à la Constitution civile du clergé, alors qu'ils proposent également des brochures constitutionnelles dans leurs catalogues ${ }^{27}$. Ces libraires se contentent de répondre à la demande, sans considération partisane, dans la continuité de la fructueuse activité d'imprimerie clandestine lyonnaise du XVIII ${ }^{e}$ siècle ${ }^{28}$. Bien qu'elle fournisse des ouvrages au clergé constitutionnel, la veuve Rusand n'en reste pas moins une militante active du clergé réfractaire, se distinguant ainsi du reste de ses confrères. Elle fait d'ailleurs l'objet de plusieurs attaques violentes dans la presse patriote locale. Le Journal de Lyon la menace notamment des pires représailles lors de la diffusion des brefs pontificaux dans la ville au cours du printemps 1791: "Madame la vendeuse de brefs, craignez qu'on ne vous brûle, vous et votre capucin ${ }^{29}$, avec vos paperasses incendiaires! $»^{30}$. En mars 1791, dans le cadre de l'enquête qui a suivi les incidents survenus dans l'église Saint-Nizier, la municipalité découvre que la veuve Rusand accueille régulièrement dans son arrière-boutique des conférences ecclésiastiques du clergé réfractaire, dirigées par l'abbé Linsolas ${ }^{31}$. Ce soutien affiché à la cause des "insoumis » ne lui cause pourtant aucun ennui judiciaire et elle poursuit ses activités éditoriales jusqu'à la fin du siège en octobre 1793.

Les militants laïcs assurent également la diffusion des textes hostiles à la Constitution civile du clergé. Dans ce domaine, la Société des demoiselles de l'abbé Linsolas semble jouer un rôle déterminant. Selon ses propres dires, Linsolas fournissait aux affiliées « plusieurs exemplaires des brochures orthodoxes opposées aux faux principes de l'Église constitutionnelle. Elles les faisaient lire aux personnes qu'on cherchait à entraîner et à celles qui avaient succombé à la séduction $»^{32}$. Le 23 juillet 1791, Anthelmine Miralier, dévideuse en soie, est arrêtée sur ordre de la municipalité pour avoir vendu des brochures réfractaires à d'autres ouvrières. Lors de son interrogatoire, elle avoue être en possession d'une

(27) Ibid., 36 L 54 : procédure contre Joseph-Sulpice Grabit (juillet 1791) ; 36 L 56 : procédure contre les libraires Maire et Mars (janvier 1792).

(28) Dominique VARrY, «Le livre clandestin à Lyon au XVIII ${ }^{\mathrm{e}}$ siècle », dans La lettre clandestine, $\mathrm{n}^{\circ} 6,1997$, p. 243-252.

(29) Allusion au R. P. Loire, qui aurait été surpris en sortant du magasin de la veuve Rusand muni d'exemplaires imprimés du bref Quod Aliquantum.

(30) Journal de Lyon ou Moniteur du département de Rhône-et-Loire, $\mathrm{n}^{\circ} 16,9$ mai 1791, p. 3.

(31) A.N., D XXIX bis 22, dossier 236 : interrogatoire de l'abbé Gayet (20 mars 1791).

(32) Jacques Linsolas, L'Église clandestine de Lyon, op. cit., vol. 2, p. 111. 
quarantaine d'imprimés. L'enquête qui suit permet de reconstituer leur circuit de diffusion. Anthelmine Miralier avait reçu les brochures des mains du cuisinier du séminaire Saint-Charles, qui lui-même les tenait des sœurs Michallet, dentellières. Interrogées, ces dernières finissent par avouer qu'elles possèdent 500 exemplaires du texte incriminé, qui leur avaient été remis par l'abbé Oléon, ancien prêtre habitué de Saint-Nizier et proche de Linsolas. On apprend également que les brochures étaient vendues neuf sols pièce, dont huit devaient être remis à l'abbé Oléon, probablement pour les frais d'impression, et un sol gardé " pour former un bénéfice au profit des prêtres persécutés $\rangle^{33}$. Le rôle de la Société des demoiselles apparaît ici à travers l'implication des sœurs Michallet, membres de l'association depuis ses débuts ${ }^{34}$. Les militantes réfractaires peuvent également faire circuler les textes hostiles à la Constitution civile du clergé par la voie du prêt. Toujours en juillet 1791, l'abbé Régny, ancien habitué de Saint-Paul, avoue à la municipalité avoir confié un lot de brochures prohibées à des ouvrières en soie de sa connaissance, qui les prêtaient à tour de rôle à leurs amies ${ }^{35}$. Le militant laïc peut également pratiquer la lecture à haute voix en public, comme ce Louis Duplâtre, domestique, arrêté par la municipalité en septembre 1792 au domicile d'une brodeuse, rue Griffon, alors qu'il lisait une brochure du clergé réfractaire devant une assemblée d'ouvrières ${ }^{36}$.

L'autre mode d'action des militants du clergé réfractaire consiste à manifester son opposition religieuse dans l'espace public. Comme pour la diffusion des brochures, les femmes semblent être très actives dans l'occupation polémique de la rue lyonnaise. Ce phénomène s'inscrit dans le temps long des émeutes urbaines de l'époque moderne, au cours desquelles les femmes jouent souvent un rôle de premier plan, par leur fonction d'information et de surveillance au niveau du quartier ${ }^{37}$. Résultant de la féminisation de plus en plus manifeste de la pratique religieuse, le fréquent engagement public des femmes en faveur du clergé réfractaire

(33) AD Rhône, 36 L 54 : enquête sur la diffusion de brochures réfractaires à Lyon (23 juillet 1791) : AM Lyon, 0306 WP 006, liasse $n^{\circ} 2$ : ibid. (23-24 juillet 1791).

(34) Jacques Linsolas, L'Église clandestine de Lyon, op. cit., vol. 2, p. 113-115.

(35) AD Rhône, 1 L 1095 : procédure contre l'abbé Régny (27 juillet 1791).

(36) AM Lyon, 2 I 2 : registre des interrogatoires des commissaires de la municipalité de Lyon, interrogatoire du 23 septembre 1792.

(37) Yves-Marie Bercé, «Les femmes dans les révoltes populaires », dans La femme à l'époque moderne (XVI $-X V I I I^{e}$ siècle), Paris, PUPS, 1985, p. 57-64 ; Arlette FARGE, «Évidentes émeutières ", dans Georges Duby et Michelle Perrot (dir.), Histoire des femmes en Occident. Tome III. XVI $-X V I I I^{e}$ siècle, Paris, Plon, 1991, p. 359-395. 
est une donnée également observable dans tout le pays ${ }^{38}$. À Lyon, comme ailleurs, les auteurs des manifestations les plus spectaculaires en faveur de l'Église réfractaire sont des femmes. Le 16 mars 1791, l'abbé Linsolas prêche le carême à Saint-Nizier, sans avoir préalablement prêté le serment comme la loi l'exige. Les jours précédents, il avait déjà prononcé un prône, dans lequel il avait appelé les fidèles à prier pour l'archevêque de Lyon. Il signifiait ainsi son refus de reconnaître la Constitution civile du clergé, qui avait supprimé le titre d'archevêque. Par la même occasion, il affichait sa volonté de ne pas tenir compte de l'élection d'Adrien Lamourette à l'épiscopat de Rhône-et-Loire, le $1^{\text {er }}$ mars précédent. Le 16 mars, des membres de la Société populaire des Amis de la Constitution sont présents au prône de Linsolas et manifestent bruyamment leur mécontentement lorsque celui-ci appelle à prier pour l'archevêque. Venues en nombre pour soutenir le prédicateur, les femmes de l'assistance - probablement membres de la Société des demoiselles - agressent physiquement les perturbateurs et l'affaire se termine par une rixe généralisée dans l'église, où l'on se bat à coups de chaises. La garde nationale doit intervenir pour séparer les combattants et évacuer l'édifice. Seuls Linsolas et deux autres prêtres sont arrêtés et l'affaire se termine par leur libération trois mois plus tard. Au-delà de l'anecdote, cet événement témoigne surtout de la capacité de mobilisation de ces militantes qui n'hésitent pas à en venir aux mains avec leurs détracteurs ${ }^{39}$. Un mois après cette affaire, toujours dans l'église Saint-Nizier, le nouvel évêque constitutionnel participe à la fête de saint Nizier, l'un des temps forts du calendrier liturgique lyonnais. Pour Adrien Lamourette, arrivé depuis peu à Lyon, cette célébration traditionnelle est l'occasion de rencontrer les fidèles et de s'inscrire dans la lignée des précédents archevêques. Dès son entrée dans l'église, il est publiquement pris à parti par deux militantes réfractaires, Madame Roberti et Mademoiselle Rondet, ex-ursuline. Ces dernières, dissimulées dans la foule, crient «Au schismatique ! Au schismatique ! »

(38) Entre autres, Claire LE FOLL, « La crise religieuse à Rouen pendant la Révolution française : gestes, attitudes et comportements féminins », dans Bernard Plongeron (dir.), Pratiques religieuses dans l'Europe révolutionnaire, op . cit., p. 331-335; Martine LAPIED, « Les Comtadines et la Révolution française : une défense prioritaire de l'identité religieuse ? », dans Gabriel Audisio (dir.), Religion et Identité. Actes du colloque d'Aix-en-Provence (octobre 1996), Aix-en-Provence, Publications de l'Université de Provence, 1998, p. 169-175.

(39) AD Rhône, 36 L54 : dossier sur le tumulte de Saint-Nizier (mars-juin 1791). Le détail de l'affaire et ses implications sont évoqués dans Paul CHOPELIN, Ville patriote et ville martyre. Une histoire religieuse de Lyon pendant la Révolution (1788-1805), thèse de doctorat, Lyon 3, 2006, vol. 1, p. 313-319. 
à l'adresse de Lamourette. Appréhendées peu après, les deux manifestantes sont toutes deux condamnées à vingt-quatre heures de prison, en dépit de l'intervention de l'évêque, qui, peu rancunier, avait demandé à la municipalité leur mise en liberté immédiate ${ }^{40}$.

Mais les femmes ne sont pas les seules à manifester publiquement leur opposition à la Constitution civile du clergé. Au séminaire SaintIrénée, les adolescents composant la classe de philosophie se rangent unanimement derrière leurs maîtres sulpiciens, qui ont refusé de prêter serment. Les prêtres constitutionnels venus remplacer les professeurs réfractaires sont confrontés à la révolte ouverte de leurs élèves, qui se retranchent dans le séminaire. Un ancien «philosophe » a laissé un intéressant témoignage sur cet épisode :

«Ce fut un hourra général [...]. Tous [les «philosophes »] repoussèrent avec énergie ceux [les nouveaux professeurs] qui nous étaient imposés. Ce fut un désordre, une bacchanale générale. On conspuait les directeurs prétendus, on fermait les classes, on se barricadait dans les chambres et quoiqu'on eût amélioré la nourriture en épuisant les provisions, elles furent mises au pillage. On brûla en grande pompe, après une procession burlesque, la chaire, dite poulailler, où on subissait les examens hebdomadaires et on la brûla avec les chandelles de la maison. Enfin, les intrus, ne pouvant plus y tenir, prièrent quelques-uns des anciens [sulpiciens] de venir rétablir l'ordre, s'il était possible. Le bon M. Molin se chargea généreusement de cette difficile tâche [...]. Aussitôt qu'il eut disparu, le plus effroyable tumulte éclata de nouveau, et tel, que les nouveaux venus se crurent obligés de faire intervenir la mairie de Lyon. Nous vîmes donc arriver M. Vitet, médecin, maire alors [...], M. Bret, procureur de la commune [...], divers agents de police membres du conseil municipal et un bataillon de gardes nationales auquel on fit charger les armes dans la cour pour nous préparer, sans doute par l'intimidation, aux exhortations patriotiques qui nous furent adressées par ces messieurs, décorés de toutes leurs écharpes, en présence des intrus rassemblés. À peine ces discours furent terminés, qu'à un signal donné, tous les pupitres furent levés et abattus à grand bruit, les boîtes d'encre, les cahiers, les livres jetés au travers de la salle, le tout avec un concert de trépignements et de sifflets.

(40) De par les Maire et officiers municipaux de la ville de Lyon. Jugement de police qui condamne la dame Roberty et la demoiselle Rondet à être détenues pendant vingt-quatre heures dans la maison de correction dite de Saint-Joseph. Du 2 mai 1791, Lyon, Aimé de La Roche, 1791, 4 p.; BM Lyon, Fonds général, ms. 805 : notes de l'abbé Duret, f 259 verso. 
La fureur éclatait sur la figure des autorités, le maire lançait des regards foudroyants. Il n'en fut que cela. Que faire contre des jeunes gens ? Depuis, les parents se hâtèrent de rappeler leurs enfants et la classe de philosophie fut fermée $»^{41}$.

Parmi ces « philosophes » résistants de février 1791, signalons la présence de Jean-Antoine Ozanam, le futur père de Frédéric ${ }^{42}$. Là encore, cette résistance collégienne à la Constitution civile du clergé n'est pas un phénomène spécifiquement lyonnais : Timothy Tackett a relevé plusieurs autres exemples de manifestations d'hostilité, parfois violentes, de la part d'élèves de collèges ou de séminaires à l'encontre de leurs nouveaux professeurs assermentés ${ }^{43}$.

Les sources consultées ne révèlent aucune action publique d'hommes adultes en faveur du clergé réfractaire. Faut-il y voir une stratégie délibérée, les femmes et les adolescents étant moins exposés à des poursuites judiciaires que les hommes ? C'est une possibilité qu'il ne faut pas rejeter de prime abord, mais aucun document ne vient corroborer cette assertion. Cet activisme pourrait également refléter la structure même des fidèles de l'Église réfractaire. De nombreux documents lyonnais - discours, rapports, mémoires, correspondances - expliquent que le clergé réfractaire recrute principalement ses fidèles parmi les femmes et les jeunes gens, tandis que les pères de familles suivent plutôt le culte constitutionnel, conduisant à la division religieuse des familles. S'il faut se garder de généraliser, comme le montrent de nombreux contre-exemples, ce constat semble néanmoins s'imposer aux yeux des contemporains et pourrait expliquer la surreprésentation féminine dans les manifestations de soutien au clergé réfractaire. Les divergences religieuses au sein de la cellule familiale, entre femmes et enfants «réfractaires» et hommes « constitutionnels », sont d'ailleurs observables dans plusieurs autres régions. C'est notamment l'un des principaux points soulignés dans le célèbre rapport Gallois-Gensonné sur la situation religieuse dans l'Ouest pendant l'été 1791. Néanmoins, les hommes ne sont pas totalement absents de l'œuvre d'assistance et de soutien au clergé réfractaire lyonnais. Les plus engagés sont des hommes jeunes, anciens «philosophes »

(41) Arch. de Saint-Sulpice., Matériaux Emery, t. III, ff $3464-3465$ : lettre de M. Dunand, ancien élève en philosophie à Saint-Irénée, à l'abbé Rony, son neveu (22 mai 1842).

(42) Gérard Cholvy, Frédéric Ozanam (1813-1853). L'engagement d'un intellectuel catholique au XIX siècle, Paris, Fayard, 2003, p. 22.

(43) Timothy Tackett, La Révolution, l'Église, la France. Le serment de 1791, Paris, Cerf, 1986, p. 195-196. 
de Saint-Irénée et peut-être membres d'une $A a$ laïque. C'est le cas de Camille Jordan, dont l'action pamphlétaire a déjà été évoquée. Son condisciple, l'avocat Auguste-Simon Ravez (1770-1849), met quant à lui ses talents oratoires au service du clergé réfractaire. Au début de l'année 1792, il défend plusieurs prêtres réfractaires déférés devant le tribunal de district pour avoir célébré le culte et baptisé clandestinement chez des particuliers. Il réussit à obtenir leur mise en liberté, non sans s'attirer l'hostilité des révolutionnaires radicaux de Lyon, qui le contraignent à quitter la ville à la fin de 1792 pour gagner Bordeaux où il effectue l'essentiel de sa carrière jusque sous la Monarchie de Juillet ${ }^{44}$. Autre ancien «philosophe » de Saint-Irénée, Mathieu-Placide Rusand assiste activement sa mère, la veuve Rusand, dans son activité éditoriale contre la Constitution civile du clergé, participant à l'impression et à la vente de brochures réfractaires ${ }^{45}$. Les autres formes de soutien masculin sont plus difficiles à déceler. La municipalité de Lyon dénonce à plusieurs reprises à l'Assemblée nationale la clémence dont ferait systématiquement preuve les juges du tribunal du district de Lyon-Ville à l'égard des prêtres réfractaires. Au regard de la documentation disponible, il est difficile de déterminer ce qui relève du soutien effectif au clergé réfractaire et de ce qui tient à la volonté de défendre le principe de liberté de conscience inscrit dans la Déclaration des droits de l'homme. Juge au tribunal de district en 1791-1792, l'ancien maire de Lyon Palerne de Savy est ainsi particulièrement attaqué par la presse patriote qui le dénonce comme un sympathisant notoire du clergé réfractaire, mais rien ne permet d'affirmer que ses convictions aient réellement influencé l'activité du tribunal.

À la fin de l'année 1791, des laïcs lyonnais décident de soutenir le clergé insermenté en organisant une «Association des citoyens catholiques de Lyon ». Il s'agit d'une fabrique privée, chargée d'organiser le culte réfractaire à Lyon, par l'acquisition d'églises vendues comme bien national, par l'achat et l'entretien du matériel liturgique, ainsi que par la rémunération des prêtres. La pétition de cette association est rejetée par le département qui la juge illégale et inconstitutionnelle. Malheureusement, l'original est perdu et l'on ne connaît ni l'identité de ces «citoyens

(44) Arch. de Saint-Sulpice, Matériaux Emery, t. III, f 3475 : témoignage de M. Chaillou, l'un des prêtres inculpés (s. d.).

(45) A. M[ELLERY], Notice biographique sur Monsieur Matthieu-Placide Rusand, ancien imprimeur du Roi, Paris, Poussielgue, 1840, p. 7-8. 
catholiques », ni le détail de leurs revendications ${ }^{46}$. Les laïcs prennent néanmoins en charge les frais de leur culte privé, en rassemblant des fonds à cet effet. La vente de certaines brochures d'opposition religieuse génère, nous l'avons vu, un petit profit pour les " prêtres persécutés ». Le Journal de Lyon dénonce de son côté l'existence d'une «Boîte à Perrette », organisée à Lyon en faveur du clergé insermenté, sur le modèle de la célèbre caisse occulte du parti janséniste à Paris au XVIII siècle. Un dénommé Orcel, demeurant place Bellecour, est même désigné comme « le ministre des finances de la gent calotine $»^{47}$. Malheureusement, les renseignements font défaut, tant sur l'organisation de cette caisse que sur l'importance des sommes récoltées.

Il est difficile de mesurer les effets de ce militantisme laïc avant le siège de 1793 . Ont-ils réussi à convaincre un nombre significatif de Lyonnais de soutenir le clergé réfractaire ? Rien n'est moins sûr. Il faut prendre avec précaution les récits postérieurs à la Révolution présentant un clergé réfractaire doté d'un large auditoire dès 1791 face à un clergé constitutionnel isolé et coupé de la population. Une étude attentive des sources contemporaines montre qu'il n'en est rien et que la ville semble bien partagée, sur ce point comme sur d'autres. Le seul effet mesurable de cet activisme concerne la diffusion à une large échelle des brefs pontificaux Quod Aliquantum et Caritas, qui conduit de nombreux prêtres de Lyon et des environs à rétracter leur serment au cours du printemps et de l'été 1791.

\section{Un activisme plus discret après la Terreur}

Il est bien évidemment légitime de se demander si ces militants laïcs ont exercé une influence sur la vie politique lyonnaise, notamment lors de la révolte fédéraliste de 1793. Là encore, aucun élément de réponse ne peut être avancé en l'état actuel des connaissances. Prêtres et fidèles de l'Église réfractaire se font très discrets après les massacres de septembre, au cours desquels trois prêtres insermentés sont exécutés à Lyon ${ }^{48}$. L'activité militante des laïcs laisse dès lors la place à une fonction de protection des prêtres réfractaires recherchés : on ne cherche plus à convaincre ou à

(46) Georges Guigue (éd.), Procès-verbaux des séances du Conseil général du département de Rhône-et-Loire (1790-1793), Trévoux, 1895, vol. 1, séance du 26 novembre 1791, p. 266.

(47) Journal de Lyon ou Moniteur du département de Rhône-et-Loire, $\mathrm{n}^{\circ} 72,14$ septembre 1791 , p. 1-2 et $\mathrm{n}^{\circ} 76,24$ septembre 1791, p. 4.

(48) Côme Simien, Les massacres de septembre 1792 à Lyon, mémoire de Master I, Université Lyon 3, 2008, 286 p. 
occuper l'espace public, mais à ménager la sécurité du culte clandestin. La discrétion est désormais de mise et le prosélytisme passe au second plan des préoccupations de ces laïcs, avant tout soucieux de perpétuer le ministère de leurs prêtres. On peut relever l'engagement d'individualités dans l'armée lyonnaise, comme Mathieu-Placide Rusand ou Camille Jordan, mais leurs motivations semblent plus politiques que religieuses. En tout état de cause, rien ne prouve que les associations laïques réfractaires en tant que telles aient joué un rôle quelconque dans les événements lyonnais du printemps et de l'été 1793.

Cette absence d'implication au grand jour peut expliquer la relative clémence des tribunaux révolutionnaires à l'égard des militants laïcs de l'Église réfractaire. Les juges de la Commission révolutionnaire de Commune-Affranchie, chargée d'épurer la société lyonnaise de ses éléments contre-révolutionnaires à partir de novembre 1793, s'attaquent surtout aux prêtres, aussi bien réfractaires que constitutionnels, accusés d'avoir favorisé la rébellion lyonnaise par leurs discours fanatiques. Les laïcs sont avant tout considérés comme des victimes du charlatanisme clérical. Il faut les guider sur la voie de la saine raison, par tous les moyens culturels mis en œuvre par les autorités révolutionnaires locales (mascarades désacralisantes, fêtes civiques, théâtre patriotique, catéchismes républicains...). Dix laïcs sont condamnés à mort et exécutés à Lyon en 1793-1794 pour avoir hébergé des prêtres réfractaires, conformément aux décrets des 29 et 30 vendémiaire an II (20-21 octobre 1793) ${ }^{49}$. Françoise Michallet, membre de la Société des demoiselles et distributrice de brochures réfractaires en 1791, est condamnée comme « fanatique, faisant dire la messe chez elle et fomentant un rassemblement de prêtres réfractaires contre-révolutionnaires ». En revanche, sa sœur n'est pas inquiétée. Plusieurs « bigotes » sont arrêtées mais échappent à la mort. Certaines sont condamnées « à la prison jusqu'à la paix », quand le tribunal commence à assouplir ses sentences au printemps 1794. D'autres sont élargies après avoir été appréhendées comme suspectes et échappent au tribunal, grâce à des témoignages de bonne moralité. C'est le cas de la veuve Rusand, personnalité emblématique du militantisme réfractaire. Elle est arrêtée fin 1793 "pour avoir vendu depuis la Révolution divers ouvrages tendant à fanatiser le peuple et à arrêter les progrès de notre liberté. L'on a regardé cette libraire comme le centre de toute la prêtraille et d'un conciliabule de royalistes et

(49) Sont exclus de ce décompte les laïcs condamnés à la fois pour leur soutien au clergé réfractaire et pour leur participation active à la défense de la ville face aux troupes de la Convention, ce dernier motif semblant déterminant dans leur condamnation à mort. 
d'aristocrates ». Sa section lui trouve néanmoins des circonstances atténuantes (« à sa décharge l'on peut dire que la citoyenne veuve Rusand a toujours beaucoup travaillé, qu'elle a élevé sa famille honnêtement et que, par la nature de son commerce qui est la dévotion, elle se trouve ruinée de ce fait ») et elle finit par être libérée ${ }^{50}$. Cet exemple démontre bien que les militants laïcs de l'Église réfractaire n'ont pas été inquiétés pour leurs idées, mais pour leur soutien éventuel à l'action de prêtres réfractaires jugés dangereux pour la cohésion nationale. Dans ce dernier cas, les laïcs tombent sous le coup de la loi. Pour les autres, les autorités préfèrent employer les armes de la pédagogie pour les remettre dans le droit chemin.

C'est cependant sous la Terreur que le militantisme laïc lyonnais trouve un nouveau champ d'action dans l'apostolat auprès des prisonniers, afin de les convertir ou de les éloigner du « schisme constitutionnel ». Si certains prêtres arrêtés exercent leur ministère dans leur lieu de détention, ils ne sont pas assez nombreux pour s'occuper de toute la population carcérale lyonnaise sous la Terreur. Les laïcs sont dès lors appelés à jouer un rôle de premier plan. Dans ses mémoires, l'abbé Linsolas évoque ce prosélytisme dans les prisons de Lyon:

« Des catholiques pleins de foi, de zèle et de charité, imitant les premiers chrétiens, allaient visiter ou faisaient visiter les prisonniers, pourvoyaient aux besoins de ceux qui n'avaient ni la facilité ni les moyens de se procurer ce qui leur était nécessaire. Ces visites étaient souvent avantageuses aux détenus, pour le spirituel, par les paroles de consolation, d'encouragement, de patience et de soumission à la volonté de Dieu qu'ils leur donnaient. Quelques-uns contribuèrent à la conversion de certains détenus, dont les uns étaient schismatiques, les autres insouciants pour leur salut $»^{51}$.

La Société des demoiselles est particulièrement mobilisée :

«Elles portaient aux religieuses détenues les lettres qu'il leur écrivait ; elles allaient les visiter souvent. Il [Linsolas] les utilisa à l'hôpital pour les femmes malades. Elles y faisaient un grand bien, soit par l'instruction chrétienne qu'elles leur donnaient, soit par les secours spirituels qu'elles

(50) AD Rhône, 42 L 101, dossier $n^{\circ}$ 113-114 : registre des prisonniers de la section de Bataillon-Affranchi (an II), fiche $\mathrm{n}^{\circ} 68$.

(51) Jacques Linsolas, L'Église clandestine de Lyon pendant la Révolution, op. cit., vol. 1, p. 150 . 
procuraient aux malades, soit par l'aumône corporelle que la société faisait. Il les utilisa pour le catéchisme qu'elles faisaient dans quelques arrondissements de la ville. On peut assurer que cette petite société rendit de grands services. Dans toutes ces bonnes œuvres, elles devaient toujours être deux associées $»^{52}$.

Parmi ces demoiselles, Linsolas distingue Marie Ravaud, qu'il dirige spirituellement depuis l'enfance et qui meurt le 20 avril 1794, à l'âge de 23 ans, après s'être épuisée dans les bonnes œuvres auprès des prisonniers. Elle devient dès lors un modèle à suivre pour toutes les sociétaires ${ }^{53}$.

L'accalmie judiciaire qui accompagne la réaction thermidorienne et les premières années du Directoire permet aux laïcs de l'Église réfractaire de renouer avec les pratiques militantes de 1791. Ils n'hésitent pas à manifester publiquement leur engagement dans les rues de Lyon et à se colleter avec leurs adversaires. Ces manifestations publiques ont certainement contribué à renforcer les craintes des autorités locales au sujet de l'agitation contre-révolutionnaire à motif religieux. Au début de mai 1795, l'abbé Glaudin, prêtre insoumis exerçant dans le quartier Saint-Jean à Lyon, signale au vicaire général Courbon qu'il a vu « un homme de la campagne, arrêté pour avoir caché un prêtre, [être] élargi et porté en triomphe par le peuple ». Au même moment, une comédie anti-catholique est copieusement sifflée au théâtre de Lyon, tandis que la plupart des boutiques de la ville sont scrupuleusement fermées le dimanche ${ }^{54}$. En mars 1796, un groupe de personnes se rassemble devant le tribunal criminel du Rhône pour apporter son soutien à des prêtres réfractaires devant comparaître dans la journée et s'oppose à d'autres citoyens venus au contraire réclamer le châtiment de ces ecclésiastiques. Les juges ont laissé une relation de l'événement, consignée dans les archives du département :

« Nous sommes rendus à la salle d'audience dudit tribunal pour examiner et prononcer sur les différentes affaires qui avoient été indiquées devoir être jugées ce jour-là. Nous étant aperçus qu'un rassemblement considérable de citoyens qui disoient hautement et confusément qu'ils étoient instruits qu'on devoit juger des prêtres, mais que si cela étoit, l'on n'en viendroit pas à bout et qu'ils les enlèveroient plutôt, que d'autres, au contraire, disoient qu'il falloit les punir et qu'ils méritoient la mort, qu'il

(52) Ibid., vol. 2, p. 33.

(53) Ibid., vol. 2, p. 115-127.

(54) Arch. segr. Vaticano, Segretaria di Stato, Emigrati della Rivoluzione francese, vol. 17, pièce n ${ }^{\circ} 327$ : lettre de Courbon à Mgr Caleppi (20 mai 1795). 
falloit les assassiner. Sur quoi, nous juges susdits, attendu l'absence d'une force armée suffisante pour comprimer ces bruits et retenir l'effervescence du rassemblement, avons, après en avoir conféré avec les citoyens commissaire et substitut du Directoire exécutif, et après avoir invité le public à garder le silence et à se tenir dans la décence convenable et due aux autorités constituées, sans pouvoir l'obtenir, renvoyé les différentes causes pour le jugement desquelles le tribunal s'étoit réuni à un autre jour $»^{55}$.

Le clergé constitutionnel devient également la cible d'attaques et de vexations de la part de certains fidèles réfractaires. En janvier 1797, des paroissiens de la Croix-Rousse viennent dénoncer, devant le juge de paix de leur canton, un projet d'attentat contre l'abbé Garnier, leur curé. Le jour de la fête de l'Épiphanie, ils découvrent que des individus avaient prévu de faire effondrer la charpente du chœur, préalablement sciée, « afin de faire écrouler l'autel, le prêtre et les assistants au moment qu'on diroit la messe ». Cette tentative d'attentat a pu être déjouée, mais la municipalité a dû faire enlever toute la partie de la charpente qui avait été sabotée et l'entreposer dans la cour de la maison municipale. Elle fait également enlever le « temple de la Raison » en bois qui était installé dans l'église et qui pouvait tout aussi bien être visé par cet acte malveillant ${ }^{56}$. En dépit de ces quelques incidents, la ville de Lyon n'est pas le théâtre d'émeutes religieuses violentes pendant le Directoire, alors qu'elles se multiplient dans les campagnes au même moment. D'autre part, la prééminence des femmes n'est plus aussi perceptible qu'en 1791-1792 : leur engagement se fait plus discret et ne se manifeste plus publiquement ${ }^{57}$. Les catholiques lyonnais restent prudents et savent jusqu'où ne pas aller afin d'éviter de nouvelles poursuites.

Sous le Directoire, le militantisme réfractaire a également pu se manifester lors des élections pour le renouvellement du second tiers des Conseils en mars 1797, en favorisant l'élection de Camille Jordan dans le Rhône. Si Linsolas et les autres vicaires généraux ont refusé de s'impliquer dans ces élections, 1'archevêque de Lyon en émigration, $\mathrm{M}^{\mathrm{gr}}$ de Marbeuf, sur la pression de Louis XVIII, a permis aux catholiques de participer au

(55) AD Rhône., 39 L 39 : procès-verbal de la séance du tribunal criminel du Rhône du 9 germinal an IV (28 mars 1796).

(56) AM Lyon, 3 WP 3 : registre des délibérations de la municipalité de la Croix-Rousse, fo 127-128 (séance du 12 fructidor an V - 29 août 1797).

(57) Ce qui est, là encore, très différent des campagnes où l'engagement féminin est toujours aussi fort dans les émeutes religieuses. Voir l'exemple de l'Yonne, développé par Suzanne Desan, Reclaiming the Sacred. Lay Religion and Popular Politics in Revolutionary France, IthacaLondres, Cornell University Press, 1990, p. 165-216. 
scrutin et de voter pour les «bons » candidats. Si Jordan a bien été élu dans le Rhône, on ne peut affirmer avec certitude qu'il a bénéficié du soutien des réseaux laïcs lyonnais, même si c'est très probable ${ }^{58}$. Le coup d'État du 18 fructidor an V et le durcissement de la législation à l'égard des prêtres insoumis obligent à nouveau les militants laïcs à abandonner l'espace public pour se consacrer uniquement à l'exercice du culte clandestin et à la protection du clergé réfractaire.

Le Concordat de 1801 met un terme à la division de l'Église catholique en France. À Lyon, la réunion des deux Églises est retardée par l'hostilité affichée par $\mathrm{M}^{\mathrm{gr}}$ de Mérinville, évêque de Chambéry et administrateur provisoire du diocèse de Lyon, à l'égard du clergé constitutionnel. Nommé au siège de Lyon en 1802, Joseph Fesch réussit, non sans mal et grâce à l'intervention directe de son neveu le premier Consul, à amalgamer les deux clergés et à rétablir la paix religieuse dans le diocèse. Les militants de l'Église réfractaire se rallient dès lors au nouvel ordre religieux. Seule une petite minorité de laïcs jansénistes refuse d'adhérer au Concordat et forme ce qu'il est convenu d'appeler la « Petite Église » de Lyon, qui a survécu jusqu'à nos jours ${ }^{59}$. Fesch doit néanmoins faire face à la résistance de quelques anciens fidèles du clergé réfractaire - principalement des femmes - qui refusent la nomination d'ex-constitutionnels dans leur paroisse. L'archevêque montre la plus grande fermeté à l'égard des « dévotes » récalcitrantes et menace d'interdit les églises dont les paroissiens empêchent l'accès au desservant nommé par l'archevêque. Les esprits s'apaisent peu à peu et les rancunes s'estompent. Les anciens militants du clergé réfractaire poursuivent néanmoins leur action dans le cadre des «congrégations» laïques lyonnaises, fondées entre 1802 et 1804 : la Congrégation des jeunes gens (1802), la Congrégation de la Sainte Famille, regroupant des pères de famille et des hommes ayant dépassé la trentaine (1802), la congrégation des dames ou Congrégation du Saint Cour de Marie (1804), la Congrégation des ouvriers (1804), la Congrégation des demoiselles, prolongation de la Société des demoiselles de Linsolas $(1805)^{60}$. L'objectif de ces associations est de participer à la reconquête

(58) Sur le clergé réfractaire lyonnais et les élections de l'an V, voir Paul Chopelin, Ville Patriote et ville martyre, op. cit., vol. 1, p. 505-507.

(59) Camille Latreille, La Petite Église de Lyon, Lyon, Lardanchet, 1911, XII-296 p.

(60) L'ouvrage de base sur le sujet reste celui d'Antoine Lestra, Histoire secrète de la Congrégation de Lyon. De la clandestinité à la fondation de la Propagation de la Foi (1801-1831), Paris, Nouvelles Éditions Latines, 1967, 368 p. Pour la fondation de ces associations et son contexte, Paul Chopelin, Ville patriote et ville martyre, op. cit., vol. 1, p. 578-586. 
religieuse de la société lyonnaise, au lendemain de la Révolution. Les mots d'ordre sont similaires à ceux de la Compagnie du Saint-Sacrement au XVII ${ }^{e}$ siècle : sanctification personnelle, par la prière et la charité, évangélisation de la société, par l'instruction et le travail, et secret à l'égard du public. De son côté, la clandestinité révolutionnaire a donné un second souffle au militantisme des jeunes laïcs, dont beaucoup se sont découverts une vocation spirituelle et souhaitent établir un réseau d'associations plus efficace. L'héritage de l'activisme catholique de la décennie révolutionnaire est surtout perceptible dans les interventions de la Congrégation des jeunes gens. Les leçons de 1791 ne sont pas oubliées sous le Consulat et l'Empire. Les membres de l'association prennent largement en main la production et la diffusion de brochures hostiles au régime impérial après l'arrestation de Pie VII, non seulement à Lyon mais également dans toute la France, contribuant à fragiliser le pouvoir de Napoléon. Les jeunes congréganistes s'efforcent surtout d'occuper physiquement l'espace urbain, notamment lors des processions, où les « impies » qui refusent d'ôter leur chapeau au passage du Saint-Sacrement sont bousculés sans ménagement. Lors des deux passages de Pie VII à Lyon en 1804 et 1805, ils fournissent une escorte remarquée au pape lors de ses déplacements. À partir de 1852, la fête du 8 décembre, avec sa procession nocturne et ses illuminations, sera la réussite la plus spectaculaire de cette occupation militante de l'espace urbain, héritée des combats de la période révolutionnaire ${ }^{61}$.

Paul CHopelin

Équipe RESEA - UMR 5190 LARHRA Université Jean Moulin Lyon 3

18 rue Chevreul 69362 Lyon Cedex 07 paulchopelin@aol.com

(61) Sur l'activité de la Congrégation de Lyon dans la première moitié du XIX siècle, JeanClaude BAUMONT, « Une association de laïques catholiques : la Congrégation de Lyon. Aspects et limites de son action lyonnaise (1817-1840) », dans Mélanges offerts à M. le Doyen André Latreille, op. cit., p. 511-532. Sur le rôle de la Congrégation dans l'établissement de la fête du 8 décembre : Jérôme CAVIGLIA, Histoire du 8 décembre. Des origines à la séparation del'Église et del 'État, Châtillon-sur-Chalaronne, La Taillanderie, 2004, 224 p. et Christophe DuBoIs, « Représenter, protester, expier : la fête du 8 décembre à Lyon (1870-1914) », dans Paul D'HoLLANDER (dir.), L'Église dans la rue. Les cérémonies extérieures du culte en France au XIX siècle, Limoges, PULIM, 2001, p. 243-255. 


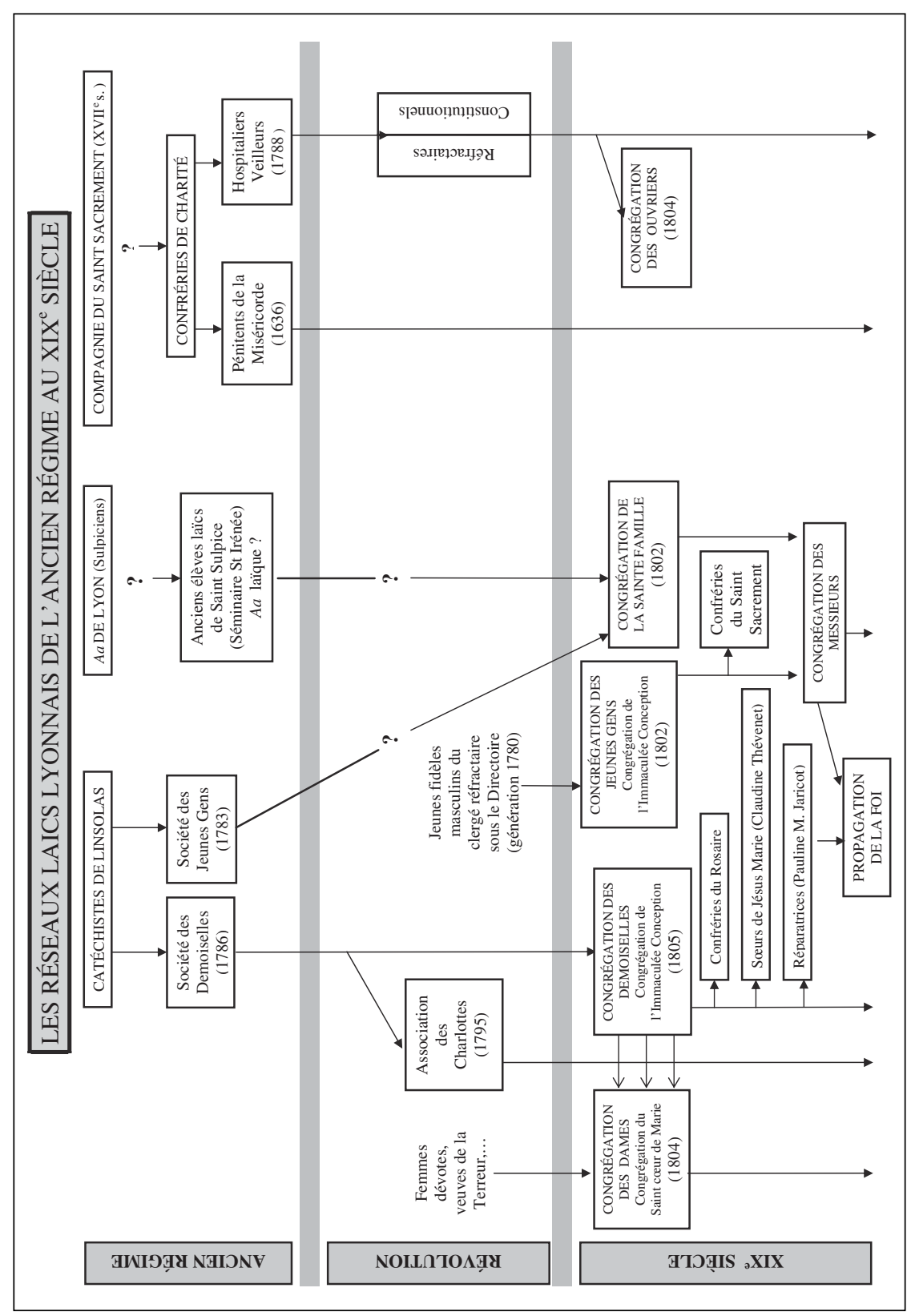

